\title{
Análisis de variables de segmentación de mercados
}

\author{
Analysis of variables in \\ the segmentation of markets
}

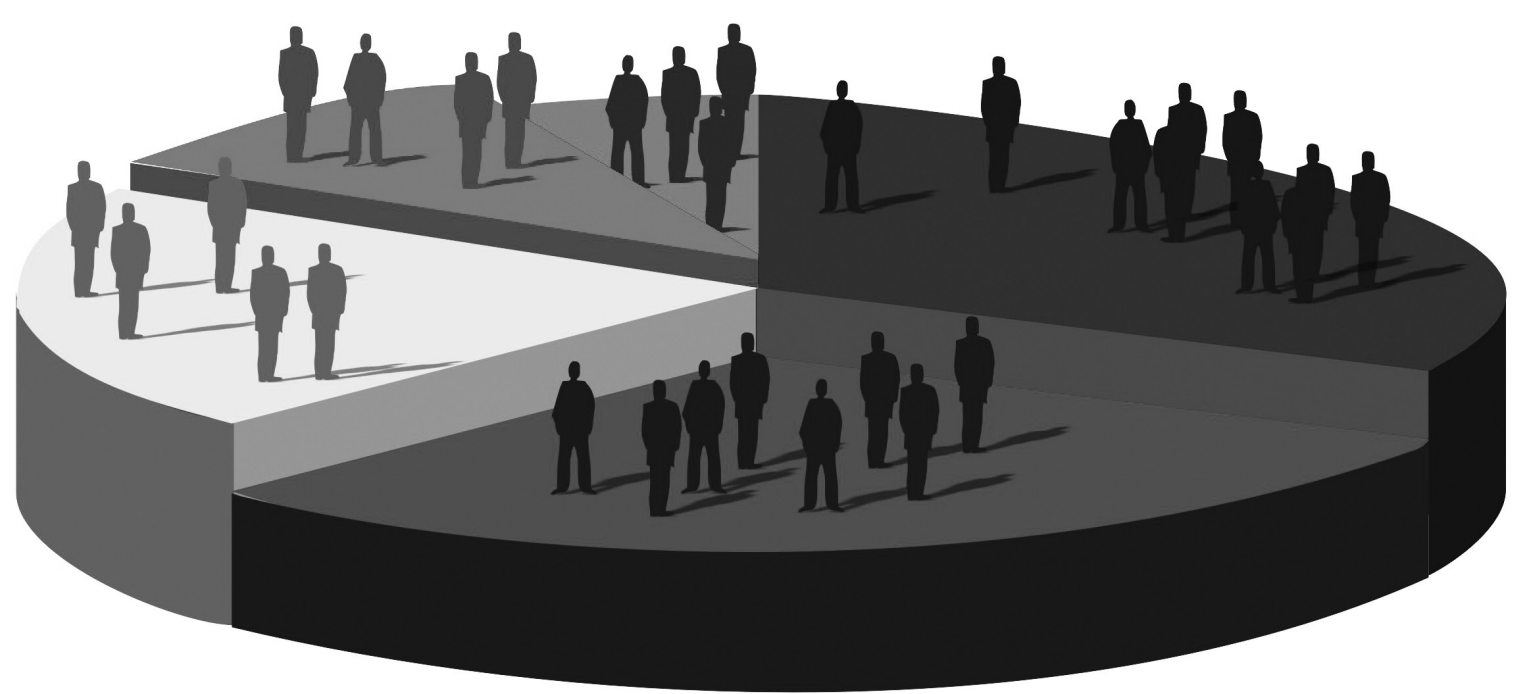




\title{
Análisis de variables de segmentación de mercados ${ }^{1}$
}

\author{
Analysis of variables in the segmentation of markets
}

\author{
Andrés Felipe Soto Orejuela ${ }^{2}$, Yeisson Diego Tamayo Salamanca ${ }^{3}$, \\ Carlos Alberto Almanza Junco ${ }^{4}$
}

Artículo recibido en agosto de 2017; artículo aceptado en octubre de 2017.

Este artículo puede compartirse bajo la Licencia Creative Commons Atribución-No Comercial-Compartir lgual 2.0 Genérica y se referencia usando el siguiente formato: Soto, A. F., Tamayo, Y. D. \& Almanza, C. A. (2018). Análisis de variables

de segmentación de mercados. I+D Revista de Investigaciones, 11(1), 78-92. DOI: https://doi.org/10.33304/revinv.v11n1-2018007

\begin{abstract}
Resumen
En la actualidad, establecer de forma progresivamente más detallada cómo se comporta el ser humano ha pasado de ser un reto analítico, basado en resultados estadísticos, a convertirse en un proceso de mayor rigor científico en dónde identificar la conducta de las personas se puede lograr conociendo más sobre el funcionamiento de su cerebro. Para satisfacer las expectativas de tan complejos consumidores, las empresas deben alinearse con la dinámica cambiante de los comportamientos presentes, para, de esta forma, mejorar los resultados en materia de la identificación de las características del consumidor que se han obtenido a través de los años. Es evidente que la evolución y la modificación de las conductas humanas influenciadas directamente por la aparición de Internet han puesto en una situación preocupante a las empresas y sus directores de mercadeo. Dar los resultados en ventas como se hacía en el pasado se ha convertido en el gran dolor de cabeza para las organizaciones; esto, sumado a la gran oferta de bienes y servicios en Internet, no permite a las empresas reaccionar a la velocidad de los gustos y preferencias de los usuarios. Las empresas, en el presente, esperan encontrar un grupo adecuado de consumidores con el fin de potenciar una marca o un grupo de productos determinado, pero su capacidad de reacción ante la velocidad con la que cambian las reglas de juego en el mercadeo empresarial, y el crecimiento desbordado de las ofertas de bienes y servicios en Internet, está poniéndoles retos ante los cuales no pueden reaccionar. En este documento se desglosan las diferentes técnicas de segmentación que ayudan a determinar con mayor precisión los procesos adecuados de segmentación, creando unas interacciones entre las diferentes herramientas de análisis para así, al final, crear una interacción general llamada segmentación sociológica geoespacial en la cual se agrupen características de estudios estadísticos especializados y análisis del comportamiento subcultural en el interior de una población específica.
\end{abstract}

Palabras clave: investigación de mercados, segmentación de mercados, tribus urbanas, millennials, consumidores, neuromarketing.

1. Revisión de literatura, tipo de enfoque cualitativo, no fue desarrollado por ningún grupo de investigación ni financiado por entidad educativa alguna. Fecha de inicio: 17 de agosto de 2016. Fecha de terminación: 16 de agosto de 2017.

2. Comunicador social, Universidad Autónoma de Occidente - Cali. MBA, Universidad Icesi. Docente de la Universidad Sergio Arboleda. ORCID ID: orcid. org/0000-0001-9898-316. Correo electrónico: Andres.soto@unicafam.edu.co.

3. Economista, Universidad de La Salle. Magíster en Dirección General, Umecit. Docente- investigador del Grupo Interdisciplinario de Investigación para la Sostenibilidad y Competitividad Empresarial de la Fundación Universitaria Cafam. Unicafam, Bogotá (Colombia), Av. carrera 68 \# 90 - 88, bloque 1, piso 6, PBX: (051) 6528600. ORCID ID: https://orcid.org/0000-0002-4266-2446. Correo electrónico institucional:Yeisson.tamayo@unicafam.edu.co.

4. Ingeniero Industrial, Universidad Católica de Colombia. Especialista en Pedagogía y Docencia Universitaria, Universidad La Gran Colombia. Magíster en Administración, Universidad Nacional de Colombia. Doctorando en Administración Universidad de Celaya, México. Docente asociado, director Consultorio Empresarial e investigador Grupo de Estudios Contemporáneos en Contabilidad Gestión y Organizaciones, Universidad Militar Nueva Granada, Km 2 de la vía Cajicá-Zipaquirá, PBX: 65000 00, Ext. 3108 - 3091. ORCID ID: https://orcid.org/0000-0002-4561-4941. Correo electrónico personal: caralmanza@gmail. com Correo electrónico institucional: carlos.almanza@unimilitar.edu.co. 


\begin{abstract}
Currently, knowing each time in a more detailed way how human beings behave has gone from being an analytical challenge based on statistical results to becoming a process of greater scientific rigor, where identifying this behavior can be achieved by knowing more about the brain function of these. In order to satisfy the expectations of such complex consumers, companies must align themselves with the changing dynamics of present behaviors, to, in this way, improve the results in terms of the identification of consumer characteristics that have been obtained over the years. It is evident that the evolution and modification of human behaviors are directly influenced by the emergence of the Internet, this has put companies and their marketing directors in a concerning situation. Giving the results in sales, as it was done in the past, has become a big headache for organizations, coupled with the great offer of goods and services on the Internet, it does not allow companies to react at the speed of tastes and user preferences. Companies currently expect to find an adequate group of consumers in order to potentialize a particular brand or group of products, but their ability to react to the speed with which they change the rules of the game in business marketing, and the overflowing growth of the offers of goods and services on the Internet, are placing challenges they cannot react to. In this paper breaks down the different segmentation techniques that help determine more accurately the appropriate processes of segmentation, creating some interactions between the different analysis tools, in order to finally create a general interaction called Sociological Geospatial Segmentation in which characteristics of statistical, specialized studies and analysis of subcultural behavior within the elements of a specific population are grouped.
\end{abstract}

Keywords: market research, market segmentation, urban tribes, millennials, consumers, neuromarketing.

\section{Introducción}

La clave del éxito de las empresas en el mercado actual se encuentra en la diferenciación de su marca o producto. El problema de ofrecer una diferenciación fácil de percibir es definir aquello que conecte con las emociones de los consumidores con los cuales se construyen los conceptos de valor percibido. En este sentido, los procesos de investigación de mercados deben ser más rigurosos; ya no basta con realizar, diseñar y formular adecuadamente las preguntas que se le van a hacer a un segmento de mercado específico. Hoy en día las empresas deben conocer los más profundos sentimientos de sus consumidores para poder lograr esa conexión que se manifiesta en la compra de un producto o servicio.

Para los responsables del mercadeo de las empresas es clave identificar los procesos humanos que se deben investigar. Es necesario superar las expectativas de la típica herramienta de investigación tipo encuesta y sus consabidos análisis estadísticos, pues, aunque los datos que se van a recoger de una muestra poblacional son importantes, si existe un mal diseño en la formulación de los cuestionamientos lo único que se van a generar son datos erróneos, lo que desemboca en una mala toma de decisiones de las organizaciones.

Décadas atrás, definir mercados, identificar segmentos o encontrar nichos de mercado era una labor más sencilla, pero hoy, ante la existencia de una subdivisión enorme en el interior de las distintas generaciones de posibles consumidores, la labor de los responsables del mercadeo empresarial es más dispendiosa, y las herramientas tradicionales de investigación de mercados no parecen ser suficientes. Esta situación, y el vacío científico existente, ha venido a ser ocupada por una nueva rama de la investigación de mercados y del mercadeo que hereda muchos de los conocimientos de los diferentes estudios realizados en Neurociencias y en particular por las Neurociencias Sociales Cognitivas, cuyos estudios se centran "en el sentido de pertenencia o no pertenencia a un grupo, que suele acompañarse de prejuicios respecto a las personas ajenas a él" (Roullet, 2015), y los estudios de Neuromarketing, que consiste en "una disciplina de avanzada, que investiga y estudia los procesos cerebrales que explican la conducta y la toma de decisiones de las personas en los campos de acción del marketing tradicional" (Bradoit, 2009). Estos nuevos enfoques vienen a sumarse a los procesos de investigación de mercados, como la segmentación de mercados tradicional y a los análisis del comportamiento subcultural, proporcionando a las empresas nuevas herramientas con las cuales comprender las motivaciones de los nuevos actores del mercado. Esta forma de entender a sus prospectos en el mercado podrá llegar a ser una labor menos inexacta y brindará a las compañías los frutos por los que tanto luchan a diario (Ariza, 2015).

\section{La segmentación de mercados desde la perspectiva tradicional}

La segmentación de mercados tradicional divide un mercado poblacional en grupos de consumidores con necesidades y características homogéneas para poder ofertarles bienes y servicios que tengan un valor de uso o de consumo. El reto está en lograr que las empresas comprendan que es necesario 
entender las particularidades de cada individuo para así llegar a satisfacerlo. Comprender que existen diferentes tipos de segmentos y que cada uno tiene sus propias motivaciones es la clave del éxito; una manera de abordar estas dinámicas es mediante el análisis descriptivo basado en herramientas cualitativas y cuantitativas.

Este tipo de estudios analizan las características de los elementos en el interior de cada segmento para que los investigadores, los emprendedores, los administradores de empresas, los mercadólogos y los publicistas diseñen planes a la medida, en función de la identificación de los rasgos de consumo. A pesar de ser el fundamento de la investigación de mercados, las herramientas que componen las técnicas cualitativas y cuantitativas al parecer no colman todas las expectativas de lo que requieren saber las empresas en el presente. Sigue existiendo un vacío en todo el proceso de la investigación del consumidor, y quizá este se deba a la veracidad de los datos que se han recopilado o a la exactitud de la información que se obtiene de las muestras; por ejemplo, el método más utilizado para obtener información del consumidor es la encuesta, que "consiste en reunir datos entrevistando a las personas. Las encuestas se pueden hacer en persona, por teléfono, por correo o por Internet. La ventaja de la encuesta es que la información viene directamente de la persona que a usted le interesa" (Stanton, 2008), y aunque la ventaja aparente de la encuesta es provenir del entrevistado, es allí donde se pueden encontrar problemas de exactitud y veracidad. Sea uno u otro el factor que esté afectando los procesos cualitativos o cuantitativos, los responsables de obtener la información del mercado deben mirar más allá de la segmentación de mercados tradicional (ver Figura 1).

Pese a que el mundo está cada vez más estandarizado por procesos de globalización, en la actualidad es un error tratar de aplicar las mismas técnicas de mercadeo a segmentos geográficamente distintos. Un notable caso lo vivió Heinz Company, que quiso utilizar la misma estrategia de mercados en dos países de Latinoamérica aparentemente iguales: Brasil y México.

En 2006, la compañía concentró su distribución en las tiendas de barrio de Brasil, estrategia que le había funcionado perfectamente en México, pero los resultados fueron negativos, pues indicaban que el $75 \%$ de las compras de abarrotes en el mercado de consumidores en Brasil era realizada en supermercados y grandes superficies y no en las tiendas de barrio, como el caso mexicano. Aunque parecería, en principio, que México y Brasil tienen características culturales y demográficas similares por el solo hecho de ser latinoamericanos, los comportamientos de los consumidores son diferentes. Estas variaciones son las que se deberían identificar al iniciar el diseño de los planes de penetración de mercados.

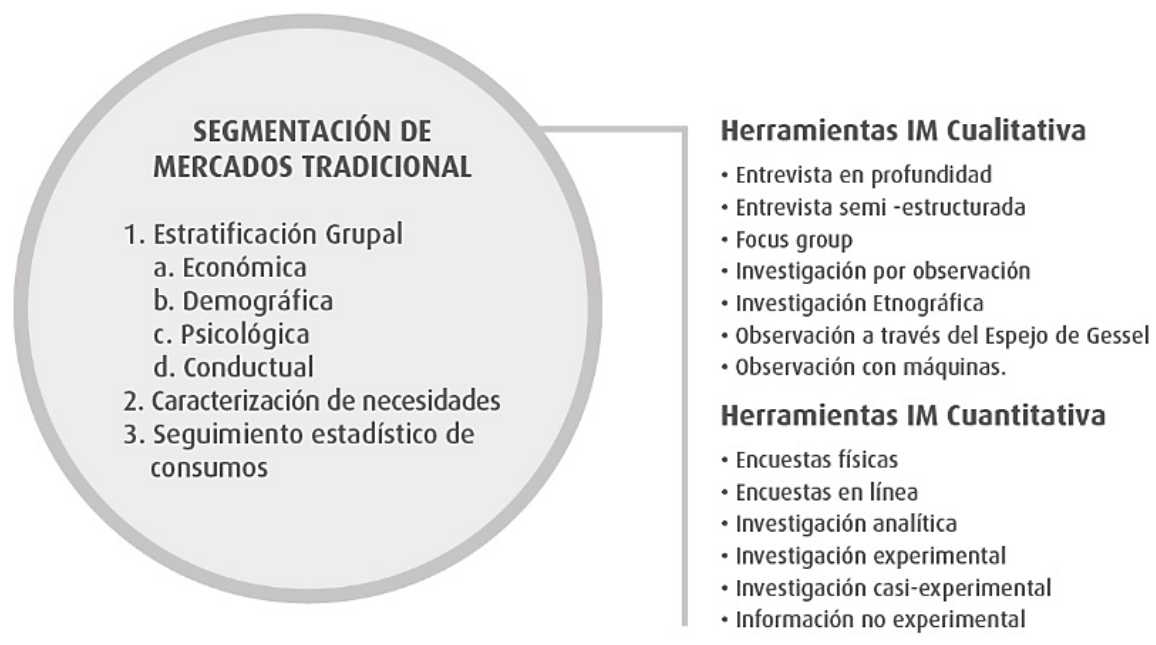

Figura 1. Segmentación de mercados tradicional. Fuente: Elaboración propia a partir de revisión bibliográfica.

Según Kotler y Armstrong (2006), las principales variables a analizar en un proceso de segmentación deben orientarse a identificar componentes de tipo geográfico, demográfico, pictográfico y conductuales (ver Tabla 1); sin embargo, una nueva dimensión emerge con fuerza en estos análisis y cobija y afecta el análisis de cada una de estas variables: las cohortes generacionales. 
Tabla 1

Características de variables de análisis en procesos de segmentación

\begin{tabular}{cc}
\hline Variable & Ejemplo \\
\hline Geográfica & $\begin{array}{c}\text { Naciones, regiones, Estados, municipios, } \\
\text { ciudades, vecindarios; densidad de } \\
\text { población (urbana, suburbana, rural), } \\
\text { clima. }\end{array}$ \\
Demográfica & $\begin{array}{c}\text { Edad, etapa del ciclo de vida, género, } \\
\text { ingresos, ocupación, educación, religión, } \\
\text { origen étnico, generación. }\end{array}$ \\
Psicográfica & $\begin{array}{c}\text { Clase social, estilo de vida, personalidad. } \\
\text { Ocasiones, beneficios, estatus de usuario, } \\
\text { tasa de utilización, estatus de lealtad. }\end{array}$ \\
\hline
\end{tabular}

Fuente: Ajustado de Kotler y Armstrong (2006).

En este sentido, al analizar el comportamiento de los consumidores es necesario tener en cuenta que existen cohortes generacionales influidas por variables geográficas, demográficas, psicográficas y conductuales. Cada una de estas dimensiones están profundamente influenciada por los diferentes sucesos contextuales e históricos que les correspondió vivir, es decir, el comportamiento de un consumidor tendrá grandes influencias de carácter político, económico, social y cultural que harán que cada generación comparta experiencias únicas y genere puntos de vista y valores comunes.

En el caso de Estados Unidos, las cohortes generacionales que definen algunos de los comportamientos típicos del siglo pasado (Generación Baby Boomers, Generación X) suman más de 190 millones de consumidores, quienes vivieron bajo la influencia de artistas o bandas como Elvis Presley, Kurt Cobain, Red Hot Chili Peppers o Metallica; esta generación fue testigo de la Segunda Guerra Mundial, la guerra de Vietnam y la llegada del hombre a la Luna, que provocaron grandes cambios en los comportamientos de consumo de los adolescentes y adultos jóvenes (Generación Y).

Lo anterior obliga a quien estéal frente de un departamento de mercadeo a que sea consciente de que lo más importante es identificar con quién está tratando, de lo contrario, ofertará en el mercado productos y servicios a quienes no corresponden. Según publicaban Philip Kotler y Gary Armstrong en el 2013 -en su libro Fundamentos de Marketing- las diferentes cohortes generacionales presentes en los Estados Unidos y que influencian a los países latinoamericanos son las siguientes (ver Tabla 2).

\section{Tabla 2}

\section{Cohortes generacionales en Estados Unidos}

\begin{tabular}{|c|c|c|c|}
\hline \multicolumn{4}{|c|}{ Perfil de las cohortes generacionales en Estados Unidos } \\
\hline $\begin{array}{c}\text { Cohorte } \\
\text { generacional }\end{array}$ & Año de nacimiento & Tamaño Aproximado & Características \\
\hline $\begin{array}{l}\text { Generación } \\
\text { Silenciosa }\end{array}$ & $1925-1945$ & 42 millones & $\begin{array}{c}\text { Desafían su edad avanzada manteniendo vidas } \\
\text { activas y adquiriendo productos y marketing que } \\
\text { les ayudará a lograrlo. }\end{array}$ \\
\hline Baby Boomers & $1946-1964$ & 76 millones & $\begin{array}{l}\text { La mayor parte está todavía en el mejor momento } \\
\text { de su ciclo de consumo; adoptan productos y } \\
\text { estilos de vida que les permitan retrotraerse en el } \\
\text { tiempo. }\end{array}$ \\
\hline Generación X & 1964-1978 & 50 millones & $\begin{array}{l}\text { A veces se considera que forman parte de una } \\
\text { brecha intergeneracional; tienden puentes entre } \\
\text { el conocimiento tecnológico de la generación Y y } \\
\text { las realidades adultas de los Baby Boomers. }\end{array}$ \\
\hline $\begin{array}{l}\text { Del tercer milenio } \\
\text { (Generación Y) }\end{array}$ & 1979-1994 & 78 millones & $\begin{array}{l}\text { Crecieron con una prosperidad relativa, } \\
\text { conectados tecnológicamente y preocupados por } \\
\text { asuntos medioambientales y sociales; también } \\
\text { tienen un fuerte sentido de independencia y una } \\
\text { percepción de inmunidad ante el marketing. }\end{array}$ \\
\hline
\end{tabular}

Fuente: Ajustado de Kotler, Armstrong, (2013). 
En los mercados actuales muchas de estas generaciones conviven de manera permanente, por lo que identificar un segmento de mercado específico causa múltiples dificultades a todo tipo de empresas. Por tal motivo, muchos de los esfuerzos comerciales, así como los mensajes que envían las marcas no están teniendo el impacto esperado y se reflexiona constantemente sobre el papel que debe estar ejerciendo la publicidad para atraer a esos públicos, pues para los compradores "un producto no es lo que un productor dice que es, sino lo que el consumidor percibe que es" (Quiñones, 2013).

Aún existen sectores empresariales que siguen centrando su estrategia de mercadeo solo en el producto y sus características, dejando de lado las experiencias entendidas como impactos sensoriales y emocionales. Este típico error empresarial "consiste en prestar mayor atención a los productos específicos que una empresa ofrece, que a los beneficios y experiencias que estos generan" (Kotler, 2013); a esto se le conoce como "miopía del marketing". En esta miopía se dejan de lado las expectativas, los sentimientos y las emociones de los consumidores, y no se toma en cuenta que el ser humano es quien determina el éxito o el fracaso de un producto mediante el uso inconsciente de dichos estímulos. Ahora bien, la ciencia se ha encargado de brindarle al mercadeo diferentes estudios de mercadeo especializados que posibilitan entender el comportamiento del cerebro de las personas ante todo tipo de estímulo, sea este un bien o servicio, una comunicación publicitaria en medios masivos o la propia experiencia con lo ofertado.

Los nuevos estudios de mercadeo especializados como las neurociencias y el neuromarketing, sumados a los estudios de segmento tradicional, les brindarían a las empresas un entendimiento de su consumidor más profundo (ver Figura 2).

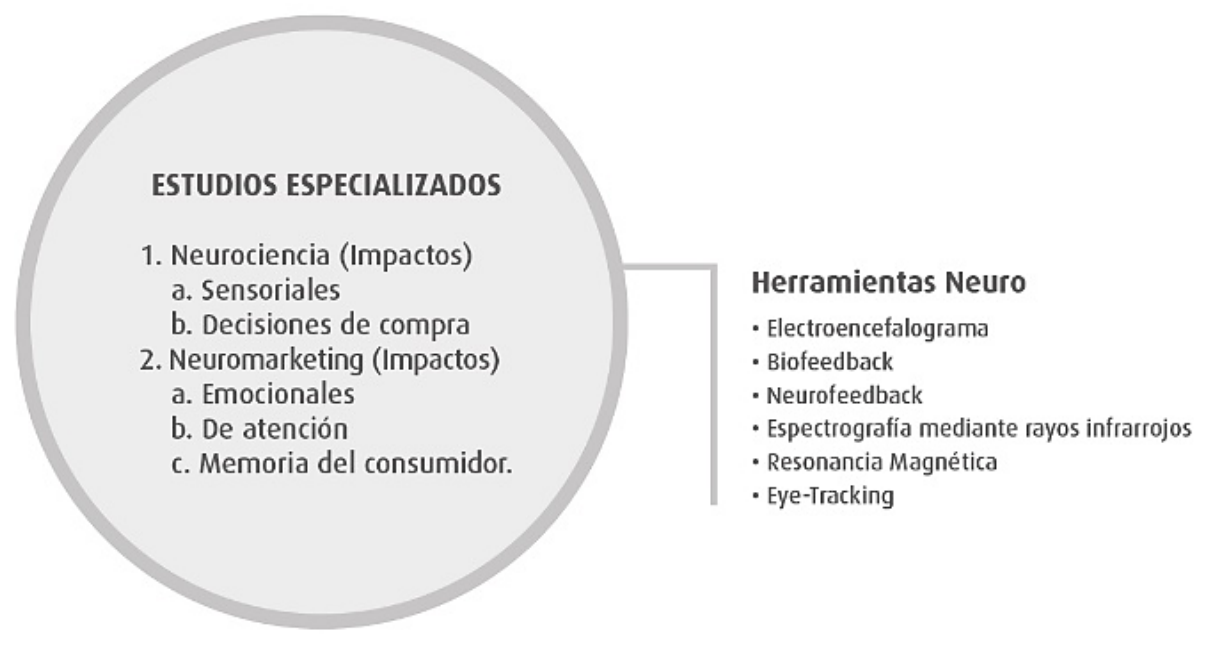

Figura 2. Estudios de mercadeo especializados. Fuente: Elaboración propia a partir de revisión bibliográfica.

Volviendo al concepto de cohortes generacionales que se ilustra en la Tabla 2, en Latinoamérica sucede algo similar al caso norteamericano, y es que, actualmente, en los países de habla hispana coexisten diversas generaciones que componen el mercado. Por ejemplo, en Colombia podemos encontrar personas pertenecientes a la "Generación Silenciosa" que son aquellos nacidos entre los años 1920 a 1940, quienes invierten muy poco dinero en compras de tipo hedónico (autoestima o autorrealización) y se limitan a realizar la mayoría de sus consumos en artículos de primera necesidad y medicina; de la generación anterior descienden aquellos que conforman "La Generación X", personas nacidas entre los años 1960 a 1980. Esta generación conforma gran parte de la fuerza laboral colombiana, que aglomera un grupo de consumidores entre los que se encuentran dueños de pyme, directores ejecutivos, gerentes, jefes de múltiples, supervisores, entre otros, que tienen un alto poder adquisitivo para comprar productos y servicios, tienen afiliaciones emocionales con distintas marcas y no temen invertir en ellas; seguidamente, se encuentran los más populares, "La Generación Y", mejor conocidos como millennials (nacidos entre los años 1980 a 2000), que son los que componen el mercado laboral, ejecutivos de 25 a 35 años que gozan de un buen poder adquisitivo, que están en proceso y cuyos consumos están enfocados en algunas marcas especializadas. Por último, están los nuevos grupos de consumidores, que los especialistas de mercadeo y publicidad han bautizado como las "generaciones $Z$ y K", jóvenes que se encuentran en las aulas universitarias y colegios, quienes serán los futuros grupos de reemplazo y nuevos actores en el mercado. 
Los consumidores sobre los que más se ha hablado en los últimos años son los denominados millennials, un grupo generacional que ha sido controversial por las posturas de algunos de sus integrantes, que crean confusión a la hora de tratar de catalogarlos. Por ello, es preciso entender que los millennials se encuentran subdivididos en subgrupos denominados "tribus urbanas", un fenómeno que es particular en esta generación, lo que ha desorientado a algunos gerentes de mercadeo en su intento por comprender un comportamiento global sobre esta generación, cuando lo que deben hacer es entender que la conducta está fragmentada.

\section{Subculturas o tribus urbanas, los millennials y las generaciones de reemplazo}

A partir del análisis del comportamiento social y cultural se pueden determinar la evolución y el análisis de las características transgeneracionales y los impactos históricos sobre un grupo o unas descendencias sociales determinadas (ver Figura 3). Autores como Kotler \& Keller (2012), en su libro Dirección de Marketing, ya hacían un fuerte llamado al respecto: "Cada cultura consta de subculturas más pequeñas, que proporcionan una identificación específica y socialización profunda con sus miembros. A su vez, las subculturas incluyen nacionalidades, religiones, grupos étnicos y las regiones geográficas. Cuando las subculturas crecen lo suficiente en tamaño y recursos, a menudo las empresas diseñan planes especializados de marketing para atenderlas".

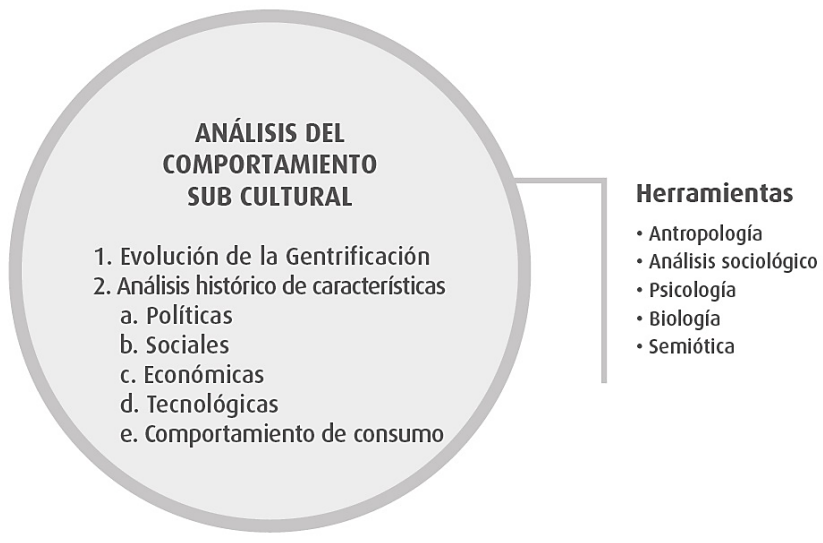

Figura 3. Estudios de análisis del comportamiento subcultural. Fuente: Elaboración propia a partir de revisión bibliográfica.

Además de la segmentación de mercados tradicional y los estudios de mercados especializados, se debe sumar esta nueva área que es el análisis del comportamiento subcultural, la cual permite tener otros puntos de vista distintos a los resultados que ofrece una encuesta. Tal es el caso de la antropología de mercados, que "estudia al ser humano, sus relevancias, miedos y tradiciones para así interpretar su constructo social y cultural. Hace un puente clave entre las relevancias de la vida del ser humano y las marcas, productos y servicios" (Klaric, 2012). Con este tipo de herramientas de estudio y análisis del comportamiento humano se pueden estudiar las subdivisiones generacionales presentes en el mercado.

La división en subgrupos tiene su mayor presencia entre los millennials, aunque no es de su exclusividad, pues ya se veían manifestaciones en las generaciones predecesoras. Pero es dentro de la "Generación Y" donde las subculturas tienen mayor presencia y dejan un camino abonado para los grupos que los vendrán a reemplazar. Los responsables del mercadeo empresarial tienen que tener la capacidad de enfrentar el estudio de sus públicos para poder determinar exactamente a quien se están dirigiendo para optimizar sus esfuerzos y no desperdiciar sus valiosos recursos. Así, con planes especializados de mercadeo, los expertos podrán utilizar las herramientas de análisis suficientes, con el fin de comprender mejor la población objetivo a la que se dirigen y entender los impulsos e intenciones de compra. Los países latinoamericanos no han sido ajenos a los diferentes movimientos sociales y recibieron una gran influencia de la cultura norteamericana y la forma en la que estaban distribuidas las diferentes generaciones con sus subculturas, produciendo casos similares. En Colombia, las primeras tribus urbanas se empezaron a manifestar en los 70; entendidas como "Un grupo de personas (tribus) conectadas entre sí, conectadas a un líder o a una idea, con un interés común y un modo de comunicarse" (Godín, 2009); entre la más representativas se encontraban una versión de los yuppies y los hippies norteamericanos, grupos que técnicamente se encontraban en el interior de los Baby Boomers y cuyos hijos serían los integrantes de la Generación X.

En nuestro continente y propiamente en el territorio colombiano, también se identificó a los integrantes de la Generación X: "El escritor Douglas Coupland los llama la Generación $\mathrm{X}$ porque están a la sombra de los boomers y carecen de características distintivas obvias" (Kotler, 2013). Los individuos de esta generación vivieron muchos cambios sociales y culturales. La mayoría nace con el aterrizaje del hombre en la Luna, crecen con la guerra fría y también fueron testigos de la caída del socialismo con el Muro de Berlín. Estos jóvenes vivieron el surgimiento de las nuevas tecnologías computacionales, disfrutaron el surgimiento de los video juegos y vieron con terror a través de los televisores a color la explosión del transbordador Challenger. Entre los fenómenos sociales surgen las primeras tribus urbanas norteamericanas 
como los rockeros, los glams y los metaleros, herederas de aquellos movimientos hippies de los 60 y 70 que adaptan su estilo de vida según los patrones de la moda que imponían agrupaciones como Kiss, Black Sabbath, $A C D C$, entre otras. Por otra parte, las comunidades afrodescendientes hallaron un lugar igualitario en la sociedad norteamericana imponiendo su estilo de vida, gustos y música, que cambió radicalmente el comportamiento de los consumidores con nuevos ritmos como el Hip Hop y el Rap. Y todos estos movimientos socioculturales darán origen a numerosas tribus urbanas como los yuppies, los geek y los nerds.

El siguiente grupo generacional son los integrantes de la "Generación Y" (los millennials o del tercer milenio), a quienes podemos definir como aquellos "nacidos entre 1977 y 2000, estos hijos de los baby boomers alcanzan los 83 millones o más, eclipsando a los miembros de la Generación $X$ y convirtiéndose en un segmento aun mayor que el de los baby boomers" (Kotler, 2013). Según la firma consultora Deloitte, se calcula que en Latinoamérica, en la actualidad, los millennials conforman el $30 \%$ de la fuerza laboral, y según sus proyecciones hacia el 2025 serán el $75 \%$.

Los millennials son, por tanto, el grupo que tiene mayor influencia en el mercado. Estos consumidores se caracterizan por ser "la primera generación de nacimiento digital, es decir, se crece con tecnología, tocando de forma virtual cada aspecto de su vida" (Caraher, 2014), de ahí que tengan mayores facilidades digitales, sean multipantalla (multidispositivos), app-dictos, críticos y exigentes, y reclamen personalización. Estos consumidores dan origen a las actuales tribus urbanas, aquellos que "crecieron en un ecosistema muy diferente al de sus padres y abuelos. No solo rodeados sino también ayudados por tecnologías, avances y ventajas que la modernidad trajo al entretenimiento, la educación y la comunicación. Comparten muchos rasgos y características que sus colegas mayores no entienden ni aprecian" (Caraher, 2014). Al igual que las generaciones predecesoras, los millennials heredaron subgrupos $\mathrm{y}$ dieron paso al surgimiento de otros. Entre las principales tribus urbanas que hacen presencia en diferentes momentos históricos están los actuales millennials tipo hípster: "la palabra hípster, sale del código callejero que comparten negros y blancos para expresar sus estados y sensaciones abstractas, ellos decían que si aceptaban el conformismo colectivo su agonía sería lenta" (Lefineau, 2011). Los integrantes de esta subcultura regularmente son bohemios que pertenecen a familias de clase mediaalta, que se radican regularmente en barrios que han sufrido procesos de gentrificación.

Otra de las principales tribus urbanas que ha sobrevivido a través de las diferentes generaciones e inició con la
Generación X son los millennials tipo yuppies "(acrónimo para "young urban professional" o joven profesional urbano), término que designa un miembro de la clase media alta, de entre 20 y 43 años de edad. El términoyuppie describe el comportamiento típico según el estereotipo del joven ejecutivo común; básicamente son personas entre 20 y 39 años, recién graduados en la Universidad, que ejercen sus profesiones y tienen ingresos medioaltos. Además, están al día tecnológicamente hablando y visten a la moda. Tienen una marcada tendencia a valorar en exceso lo material, siendo típicas las inversiones en bolsas de valores, la compra de autos y el mantenerse a la vanguardia en tecnología (móviles más sofisticados, digital notepads, etc.). En Latinoamérica, el fenómeno yuppie se presenta en las clases sociales emergentes y su estilo de vida se percibe como la meta a alcanzar, cuya promesa es satisfacer necesidades primarias, necesidades de autoestima y autorrealización $y$, finalmente, llegar a un nivel socioeconómico superior y distante del de sus padres. El yuppie latinoamericano se encuentra en una gran encrucijada: primero, actuar como sus similares norteamericanos y europeos, aunque no entienda cómo son, ni cómo se comportan, quizá basándose en la información que los medios masivos ofrecen como películas, series de televisión, programas de entretenimiento, entre otros. Por otra parte, lo que pretenden es mejorar su situación socioeconómica para escalar socialmente a través de una carrera universitaria con la promesa de obtener un gran puesto.

Otra de las tribus emergentes que tiene presencia en este grupo generacional son los millennials tipo muppies, quienes quieren desplazar a los hípsters con su nuevo estilo de vida y costumbres. Estos jóvenes son nativos digitales que tienen como única preocupación llevar una vida sana, libre de cigarrillo, alcohol y cualquier tipo de drogas. Heredan su búsqueda de alcanzar el éxito de los yuppies de los 80; ya no aspiran a pertenecer a una multinacional, sino que están fundando su propia startup o pequeña empresa o quizás en el momento ya pertenecen a alguna.

Otra subcultura son los millennials tipo dinky (dink o también dinki), término que significa en inglés double income, not kids yet (doble sueldo, ningún niño por ahora), el cual "fue acuñado por el sociólogo Millbard Brown en el Reino Unido, en la década de los 80, y se aplica a un segmento de población cada vez más grande, compuesto por parejas jóvenes o matrimonios de 25 años en adelante, que renuncian a la paternidad en aras de ascender personal y profesionalmente. Lejos de los gastos que representan pañales, jardín, colegio y universidad, estas parejas prefieren darse una vida de lujos, viajes, buenos restaurantes, tecnología, maestrías, doctorados (El País, 2014). 
Los dinkies consideran que el hecho de tener hijos hará que sus ingresos se vean afectados directamente, por ello prefieren postergar el matrimonio y la familia para invertir en ellos mismos, por ejemplo "los hombres solteros gastan más que los casados en productos como bebidas alcohólicas, automóviles nuevos y educación. En comparación con las mujeres casadas, las solteras tienden a gastar más en automóviles nuevos, entretenimiento y adquisición de casa para vivir en una zona segura" (Hoyer, 2015).

Frente a esta tendencia, el economista Kenneth Elzinga argumenta que "en las sociedades ricas los niños vienen a ser bienes inferiores, o sea que a mayor ingreso menor demanda de dicho bien. En cambio, en sociedades pobres, a menor ingreso hay más demanda de niños, debido a la falsa ilusión de que más hijos podrían traer mayores ingresos a casa" (Perez, 2010).

Al igual que con los grupos abordados previamente, existen numerosas subdivisiones más de los millennials que hacen presencia en el mercado, entrelos que se encuentran:swaggers, ravers, clubers, kogals (yamambas, ganguros, ganjiro, spotsu gyaru, spots gal, onegyaru, onessangal, kogal panda), cyberpunks, hackers, decorers, freegans skatersmods, grunges, rockeros o rocanroleros, metaleros (heavy metal, trash metal, death metal, black metal), góticos, rastafaris, emos, cools, andróginos, metrosexuales, retrosexuales, urbersexuales, tecnosexuales, lumbersexuales, spornosexuales, punks, skinhead, barras bravas de futbol, freaks, animalistas, automovilistas, adeptos a la ciencia ficción, coleccionistas, deportistas, historiadores, etc. También hay que dar una mirada a los comportamientos de la última etapa de los millennials, que son aquellos que nacen y crecen con una gran influencia del mundo digital, que pueden ser subcategorías de los muppies o hípster como: los youtubers, gamers, booktubers, entre otros.

La siguiente generación, aquella que hace presencia en la actualidad en las aulas de clase universitaria y en los últimos grados de estudios secundarios de colegio, se denomina "Generación Z o Centennials", que ya aparecen identificados en el último estudio de The Nielsen Global Generational Lifestyles Survey realizado en 2015 y en los últimos años se viene tratando de teorizar sobre ellos. De "acuerdo con la firma de investigación de medios Kantar Ibope Media, llegaron al mundo a partir de 1997 con un smartphone o tablet debajo del brazo y con una sobreexposición a la información y a la era digital jamás vista" (Semana, 2017). Los intereses se concentran en ser socialmente aceptados por una comunidad que hace presencia en la red, en donde las aspiraciones de algunos es lograr el gran reconocimiento y popularidad a toda costa, convertirse en YouTubers; ser realizadores audiovisuales y producir Smart films; ocupar mejores posiciones laborales que los millennials, pues ellos no temen al mundo tecnológico y se relacionan mejor con él; provocar cambios sociales, viajar o desarrollar estudios en el exterior y disfrutar las bondades que les brinda la naturaleza. Estos jóvenes "comienzan a salir de las universidades, a incorporarse al mundo laboral y a reclamar su sitio en el mundo. Al igual de lo que ocurrió con la Generación $X$ y lo que está aconteciendo con los millennials, la llegada de los jóvenes $Z$ a la edad adulta tendrá un fuerte impacto en la sociedad y las empresas" (Ortega, Soto \& Cerdán, 2016).

Herederos de comportamientos de las generaciones predecesoras se halla la evolución de una clase de yuppie denominada yetties. El término es un acrónimo que significa young entrepreneurial technology based, es decir, jóvenes empresarios o emprendedores que basan su estilo de vida en el mundo tecnológico. Es usual que apenas se gradúen de la universidad los yetties conserven su estilo de vida y forma de vestir, y sorprende verlos en una reunión de negocios vestidos con yin, quizá usando una chaqueta de cuero, calzando tenis, una mochila en donde llevan sus accesorios tecnológicos y los de su medio de transporte preferido, que es la 'bici', entre otras particularidades como que no "cumplen horarios sino objetivos: su semana laboral tiene más de cinco días y el tiempo libre es escaso. Suelen postergar lo familiar por los negocios. Viajan por todo el mundo vendiendo sus empresas. Y quieren ser ricos lo más rápido posible" (Libedinsky, 2000).

Pero, el dilema para los profesionales del mercadeo no es lograr identificarlos, el verdadero dilema es que la brecha entre los $Z$ y quienes vienen detrás de ellos es muy corta. En esa distancia se agrupa la que se considera la nueva generación, conformada por niños de cero años hasta preadolescentes de 15 años, último rango en donde Nielsen había llegado en su clasificación en el 2015. A este último grupo lo han empezado a denominar la "Generación K", término que se ha venido acuñando por tener como principal referente entre los grupos de preadolescentes a Katniss Everdeen, personaje de la saga "Juegos del Hambre". Estos jóvenes son considerados jóvenes ansiosos, entre los cuales el "72\% de los adolescentes se preocupa por el terrorismo, un 64\% por el cambio climático, un $79 \%$ sufre ante la idea de conseguir empleo y un $72 \%$ sobre las deudas potenciales que un día tendrán que pagar. A eso hay que sumar que son solitarios y se sienten así. Aunque están constantemente conectados a la red, son solitarios y por ello valoran como algo Premium el poder estar físicamente con la gente" (Puromarketing, 2016). Aunque tanto la Generación Z como la Generación $\mathrm{K}$ son los grupos actuales de consumidores que están entrando a jugar su rol en el mercado, existe poco material científico para definirlos detalladamente, pero hay que tener los ojos bien puestos hacia el futuro pues 
son las generaciones que reemplazarán los espacios que están dejando los Baby Boomers y la Generación X.

\section{¿Cuál será la nueva frontera?}

La segmentación de mercados tendrá que convertirse en una tarea de mayor rigor. A medida que el ser humano cambie y modifique sus comportamientos, los administradores de empresas, emprendedores, mercadólogos y publicistas deberán replantear la forma como estudian a su consumidor. Mejorar en los procesos de investigación de mercados dará como resultado conocer cómo se conectan los consumidores con sus marcas, lo que facilita la creación de valor percibido en los consumidores; esta es la razón por la que "el secreto del consumo no está únicamente en el producto, sino en cómo este es procesado, percibido, imaginado o fantaseado por el consumidor" (Quiñones, 2013).

La nueva frontera que se propone para abordar los estudios de investigación de mercados y análisis del comportamiento del consumidor se basa en la utilización de un modelo denominado Segmentación Sociológica Geoespacial (SSG), que pretende identificar de una forma rigurosa la manera en la cual las empresas crean valor para sus usuarios (ver Figura 4). Dicho modelo propone integrar las herramientas utilizadas en la segmentación de mercados tradicional, los estudios especializados y el análisis del comportamiento subcultural para encontrar variables que midan de forma más viable la conducta del consumidor y de este modo sea un aporte a las empresas para que así oferten mejores propuestas de valor.

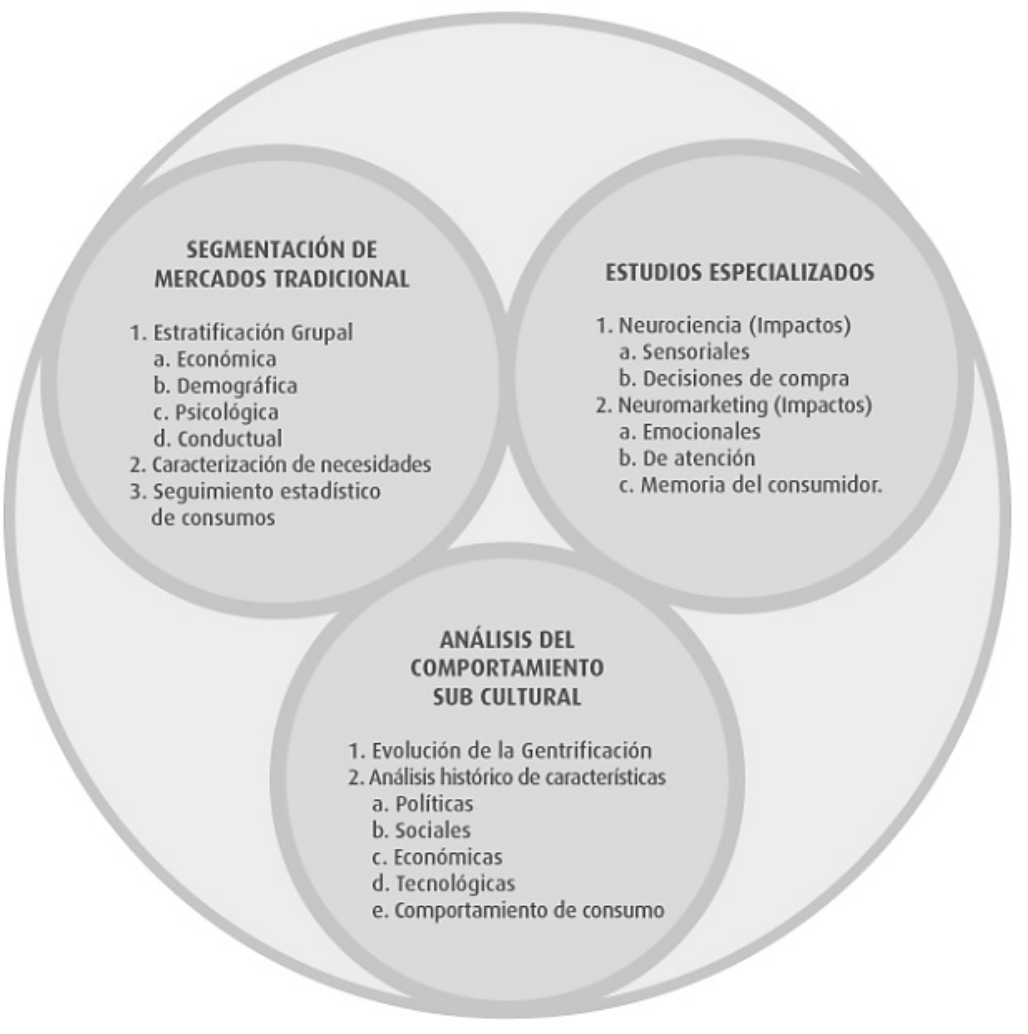

Figura 4. Estudios de mercado encaminados a la creación de valores. Fuente: Elaboración propia a partir de revisión bibliográfica.

En este sentido, se debe entender que la segmentación del consumidor es multidimensional y cambia continuamente frente a la evolución del comportamiento subcultural, puesto que hoy en día las personas están buscando comprar productos innovadores y esto significa "ofrecer valor agregado a las personas a través de los productos o servicios que les vendemos" (Klaric, 2014).
En la actualidad, se siguen utilizando los métodos tradicionales de investigación de mercados donde se cuentan los estudios cualitativas y cuantitativas. De los estudios cuantitativos las empresas vienen utilizando contratistas que les brindan servicios como la aplicación de encuestas físicas; otras, tienen el personal y equipos para desarrollar los focus group (grupos focales), 
entrevistas en profundidad, desarrollos de observación etnográfica, entre otros, y los resultados siguen siendo los mismos. En ocasiones, la exactitud y la veracidad de los datos recopilados ofrecen un grado de incertidumbre para la toma de decisiones. De otra parte, ha surgido otra manera de obtener datos secundarios de los usuarios, datos que "son información que ya se había recopilado para otro problema o asunto". Es decir, ya "está ahí", entre otros lugares, en el ciberespacio (Hair, Bush, \& Ortinau, 2010) y gracias a los nuevos desarrollos que brinda la Internet las empresas pueden utilizarla para mejorar sus propuestas de valor. Además, en el presente existen nuevas herramientas de investigación que se encuentran en la red y que ofrecen a los investigadores de mercados una manera más económica y a veces gratuita para encontrar datos del consumidor, como por ejemplo: Google Forms, Google Analytics, Facebook Stadistics, monkeySurvey.com, onlineencuesta.com, salesforce.com, entre otras (Soto \& Rios, 2016).

Las empresas no pueden solo afiliarse a las herramientas tradicionales para entender al consumidor, hoy deben aprovechar todos los recursos que los estudios especializados pueden aportar. Las compañías deberán buscar la forma adecuada de integrar a sus procesos el uso de ambos tipos de gestión de investigación, en tanto los "proveedores y servicios de investigación de mercados suministran la mayor parte de la información requerida para tomar decisiones de marketing" (Malhotra, 2008), decisiones que pueden llevar a la aplicación de nuevas metodologías de investigación como son la neuropsicología, las neurociencias y la antropología sensorial o el neuromarketing, que facilitan la comprensión de las necesidades de los consumidores y se encuentran codificadas en el subconsciente, porque "la única manera efectiva de entender lo que la gente verdaderamente quiere decir es ignorar lo que dicen" (Rapaille, 2007). Por ello, es necesario abordar los estudios del consumidor actual con la combinación de herramientas que les permitan a las empresas superar los potenciales errores que arrojan los datos atípicos de la estratificación grupal, el desconocimiento del comportamiento típico subcultural de la población objetivo o los impactos sensoriales o emocionales que atraviesan los compradores a la hora de realizar la transacción comercial.

La interacción entre el estudio de segmentación de mercado tradicional y los estudios especializados se denomina análisis neuropsicológico (NP), el cual busca determinar los efectos que se pueden producir en los procesos cognitivos, psicológicos y emocionales del comportamiento individual (ver Figura 5), basado en los aportes que los estudios cuantitativos y cualitativos, sumados a los de la neurociencia y neuromarketing, le brindan al investigador para entender, de una forma más especializada, a un grupo generacional y sus subdivisiones.

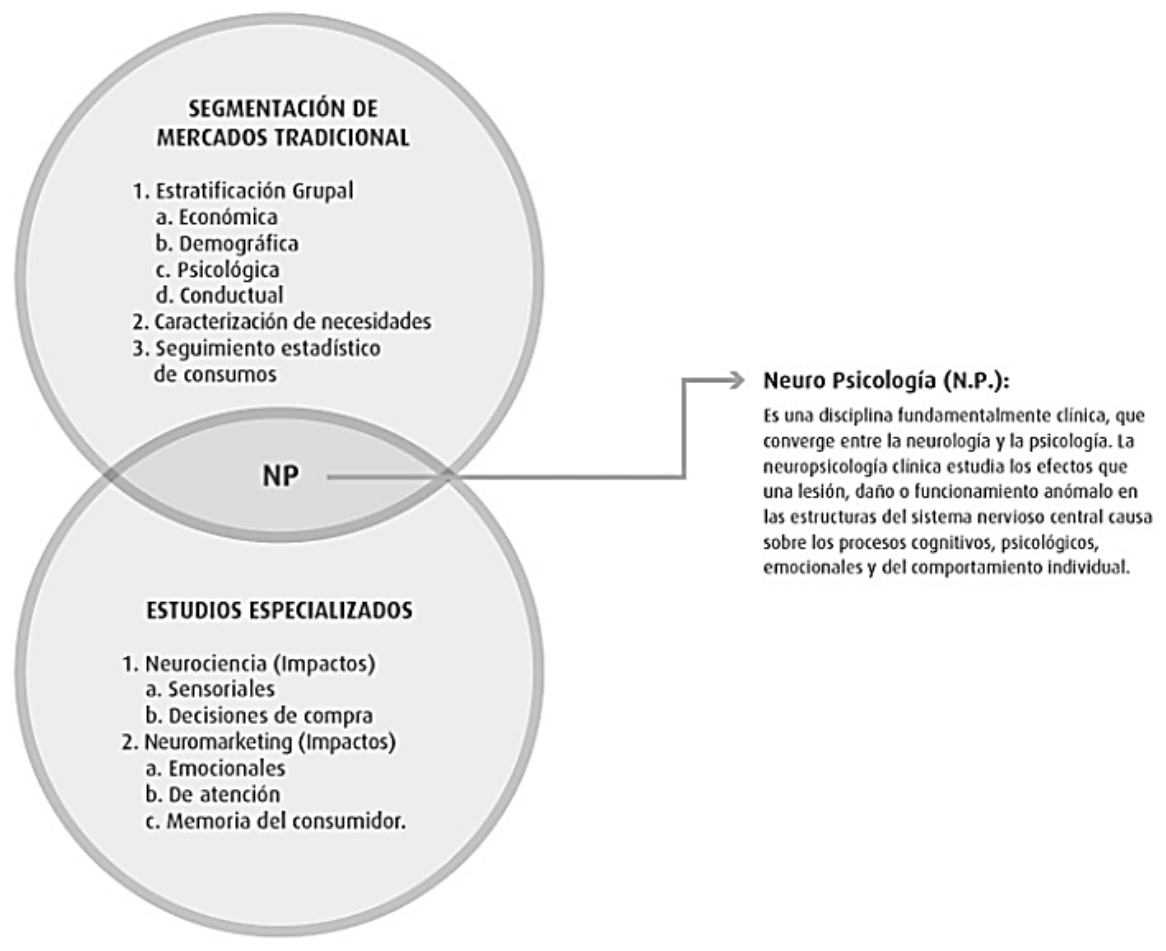

Figura 5. Interacción neuropsicológica (NP). Fuente: Elaboración propia a partir de revisión bibliográfica. 
Pero el modelo propone ir más allá de la sola interacción de la segmentación tradicional y los estudios especializados. El modelo SSG también plantea una interacción entre los estudios especializados y otra rama de estudios, que es el análisis del comportamiento subcultural; a esta interacción se le va a denominar neurobiología de desarrollo (NBD), la cual analiza las particularidades del comportamiento histórico de un integrante particular de un grupo social, a partir del conocimiento previo de sus características cognitivas y perceptuales, con el fin de determinar los posibles deseos y necesidades de una población objetivo (ver Figura 6).

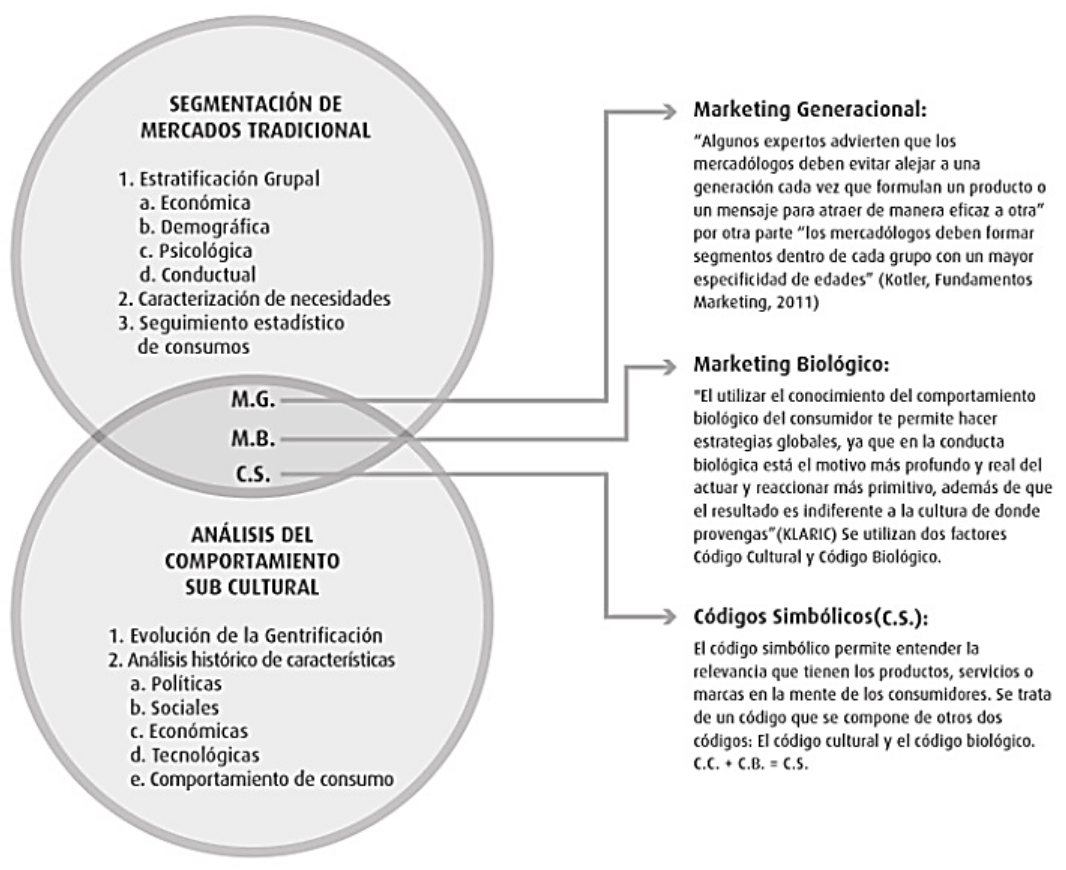

Figura 6. Interacción neurobiológica de desarrollo (NBD). Fuente: Elaboración propia a partir de revisión bibliográfica.

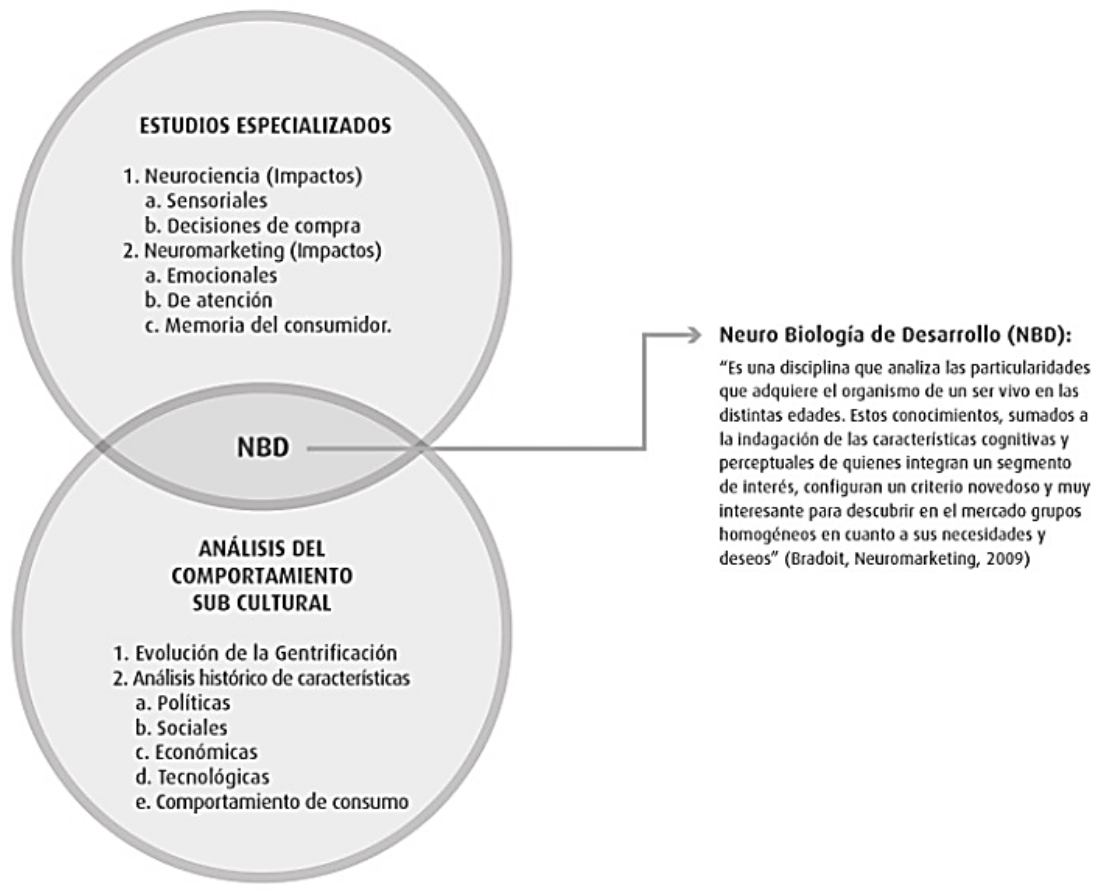

Figura 7. Interacciones entre el marketing generacional (mg), el marketing biológico $(\mathrm{mb})$ y los códigos simbólicos. Fuente: Elaboración propia a partir de revisión bibliográfica. 
Por otra parte, el modelo SSG también propone otra interacción entre la segmentación tradicional de mercados y el análisis del comportamiento subcultural, que da como resultado el uso de tres herramientas: el marketing generacional, el cual busca realizar un proceso de mercadeo integrando varias generaciones; el marketing biológico, que busca utilizar los datos históricos de consumo con el fin de diseñar estrategias específicas por grupos sociales y los códigos simbólicos, los cuales permiten entender las relevancias específicas de cada producto en el interior de un grupo social determinado (ver Figura 7).

Como resultado final a partir del análisis de la bibliografía encontrada y el desarrollo del proceso de investigación, se propone la creación de una nueva herramienta de análisis de mercado llamada segmentación sociológica geoespacial (SSG), la cual utiliza como herramienta de estudio la segmentación tradicional de mercados, para identificar cada uno de los segmentos teniendo en cuenta los grupos sociales, el uso de estudios especializados por los impactos sensoriales y emocionales que afronta el consumidor en el instante de la compra y el análisis del comportamiento subcultural a partir del estudio de la evolución de la gentrificación social. Y el uso de las respectivas intersecciones: la NP, por el análisis del comportamiento histórico de los integrantes de una sociedad en particular; el NBD, por la determinación de los posibles deseos y necesidades de una población; el análisis del MG, por la integración de las generaciones; el MB, por el análisis de los datos históricos de consumo y el uso de CS, por las relevancias del uso y consumo de cada servicio o producto (ver Figura 8).

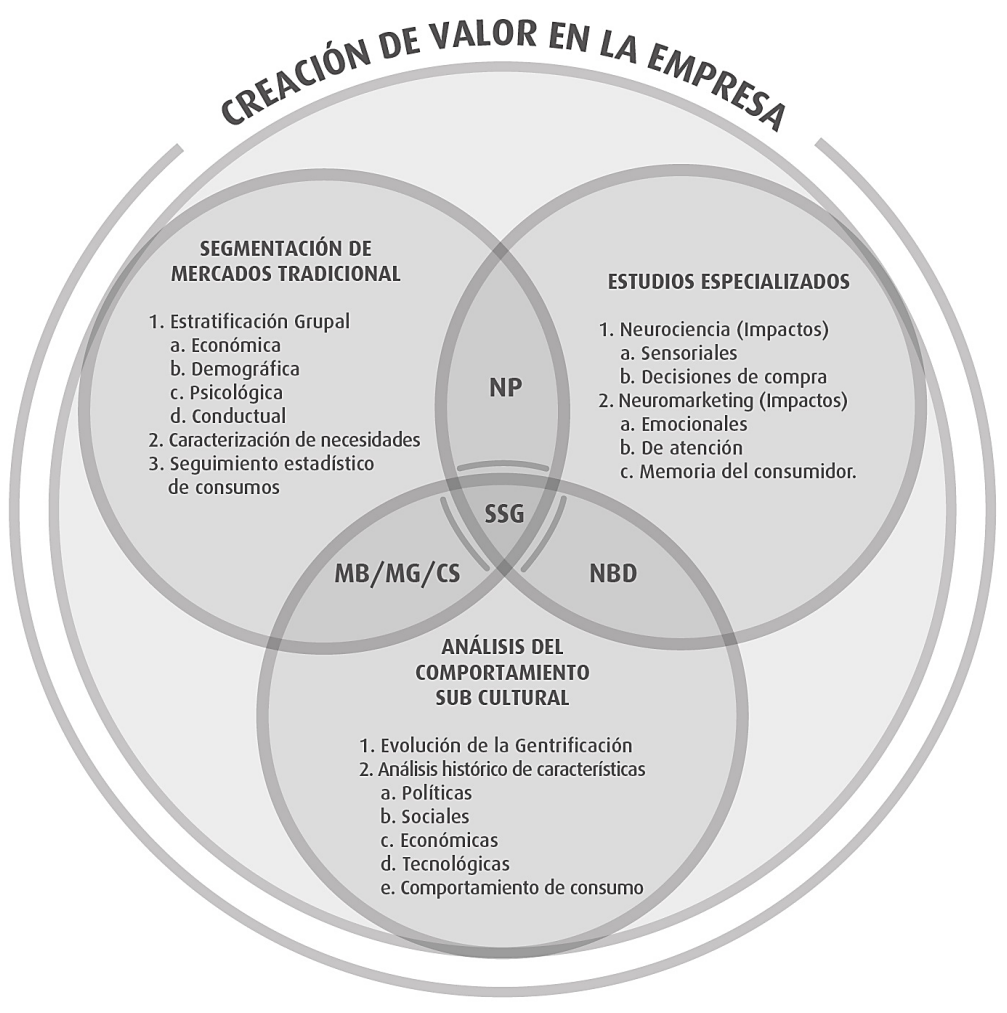

Figura 8. Identificación de la segmentación sociológica geoespacial (SSG). Fuente: Elaboración propia.

La segmentación sociológica geoespacial busca determinar el análisis específico por grupo social, estableciendo las características y evoluciones de consumo que determinan los cambios necesarios en los productos, bienes o servicios que se ofrecen a una sociedad determinada.

\section{Resultados}

Se puede llegar a afirmar que un esquema como el de segmentación sociológica geoespacial es una herramienta que facilita la parametrización de la segmentación de mercados en la actualidad, debido a que se tienen en cuenta diferentes herramientas de análisis demográficos, económicos y sociales, como el estudio de los impactos emocionales o sensoriales (si es posible según el producto) y el análisis de los procesos de la gentrificación y su impacto en el consumo de productos específicos.

Un gerente de mercadeo debe comprender que la dinámica del mercado en la actualidad varía a una 
velocidad acelerada $y$, en ocasiones, las empresas no alcanzan a reaccionar. Los hábitos, costumbres y estilos de vida de los consumidores están provocando que los rangos generacionales sean más cortos, y las herencias tribales de generación en generación mutan, afectando de forma directa la manera en la cual se relacionan con los productos y las experiencias que estos le brindan.

La migración de los usuarios hacia otros mercados sustitutos o de commodities debido al desconocimiento del nuevo consumidor es el principal error que se comete, y lo que debe hacer una empresa es alinear su misión y su visión a las nuevas condiciones del mercado para que los expertos en mercadeo reaccionen de manera más efectiva.

La implementación, en el interior de las empresas, de los estudios de segmentación sociológica geoespacial generará un cambio del modelo mental de los actuales gerentes en las organizaciones, pues estos, permanentemente, están demandando resultados en las ventas de bienes y servicios. Pero en ocasiones la propia miopía mercadológica de las empresas les hace perder la claridad sobre las características de sus consumidores. En la actualidad, para conseguir un incremento en los posibles seguidores de una marca o producto los llamados lovemakers - las empresas tienen que brindarles a sus usuarios las mejores experiencias con sus marcas, pues de lo contrario solo se puede esperar el fracaso. Identificando las conductas de los prospectos y la forma como se relacionan con su entorno, un gerente puede mejorar e implementar cambios sugeridos en la marca o productos y servicios (cambios como símbolos, colores, formas, imágenes, precios, cantidades, calidades, promociones, días de compra, etc.), una forma de crear cercanía con la marca, lo que puede llegar a influenciar de forma directa el consumo de un determinado grupo social, el consumo de una marca o el consumo de un producto en especial.

Para finalizar, la implementación de la segmentación sociológica geoespacial (SSG) les brindará a las organizaciones una visión diferente a la de mantenerse en el mercado y tener éxito, pues el principal deber de las empresas es orientarse hacia las expectativas del cliente, y esto solo se puede lograr identificando claramente el grupo social, entre los que se encuentran aquellos tribalizados. Que una organización pueda identificar una tribu puede brindarle beneficios, porque estas serán los canales de comunicación más efectivos, pero no están a la venta ni se alquilan. Las tribus no hacen lo que tú quieres, hacen lo que ellas quieren. Razón por la cual reunir una tribu y liderarla es una inversión tan poderosa en marketing (Godin, 2009). Una inversión que va a determinar el éxito o fracaso de una marca.

\section{Referencias}

Ariza, H. (2015). Revisitando estrategias de sostenibilidad de las empresas a través de una visión sistémica empresarial. I+ D Revista de Investigaciones, 5(1), 23-42. Recuperado de https://doi.org/10.33304/revinv.v05n1-2015002

Bradoit, N. (2009). Neuromarketing. ¿Por qué tus clientes se acuestan con otro si dicen que les gustas tú? Barcelona: Gestión 2000, Planeta de Agostini.

Caraher, L. (2014). Millennials en la oficina, cómo lidiar con una generación que no sigue las reglas. Bogotá: Planeta Colombiana S. A.

El País (2014). La vida de los Dinky: parejas que no aspiran a tener hijos. Entretenimiento. elpaís.com. co. Recuperado 5 de mayo de 2017, de http://www. elpais.com.co/ entretenimiento/la-vida-de-los-dinkyparejas-que-noaspiran-a-tener-hijos.html

Godin, S. (2009). Tribus, necesitamos que tú nos líderes. Barcelona: Gestión 2000.

Hair, J., Bush, R. \& Ortinau, D. (2010). Investigación de mercados en un ambiente de información digital: Mcgraw-Hill/Interamericana Editores, S. A.

Hoyer,W., MacInnis, D.\&Pieters, R. (2015). Comportamiento del Consumidor: Cengage Learning.

Klaric, J. (2012). Estamos Ciegos. Bogotá: Planeta Colombiana S.A.

Klaric, J. (2014). Véndele a la mente y no a la gente, neuroventas. Perú: Nomo impresores.

Kotler, P., \& Armstrong, G. (2006). Principles of marketing management. New Delhi: Hall of India private limited.

Kotler, P. \& Gary, A. (2013). Fundamentos de Marketing. México: Pearson Educación México.

Kotler, P. y Keller, K. (2012). Dirección de Marketing. México. Pearson Educación México, S. A. de C.V.

Lefineau, M. (2011). Tribus urbanas, la indumentaria desde la perspectiva cultural. Bogotá: Ediciones de la U.

Libedinsky, J. (2000). Los nuevos yuppies se llaman yetties. La Nación. Recuperado 20 de agosto de 2017, Recuperado de http://www.lanacion.com.ar/9626los-nuevosyuppies-se-llaman-yetties

Malhotra, N. (2008). Investigación de mercados. México: Pearson Educación México, S. A.

Ortega, l., Soto, I. \& Cerdán, C. (2016). Generación Z, El último salto generacional. Atrevia, la consultora global de comunicación con espíritu latino. España: Deusto Business School, Universidad Deusto.

Perez, A. (2010). Los Dinkys: Elección de no tener hijos. Recuperado dehttp://www.entrelineassdo.com/2017/07/ los-dinkys-eleccion-de-no-tener-hijos.html

Puromarketing (2016). Generación K: Los adolescentes que más difícil se lo pondrán a las marcas. Recuperado 20 de marzo de 2017, de http://www.puromarketing. com/88/26994/generacionadolescentes-mas-dificilpondrana-marcas.html 
Quiñones, C. (2013). Desnudando la Mente del Consumidor, Consumer Insigths en el Marketing. Perú: Planeta Perú S.A.

Rapaille, C. (2007). El Código Cultural. Bogotá: Grupo Editorial Norma.

Roullet, B. (2015). Neuromarketing. México D. F.: Trillas.

Semana. (2017). Millennials y Centennials, las generaciones que cambiarán el mundo. Recuperado 11 de julio de 2017, de http:// www.semana.com/educacion/ articulo/millennials-ycentennials-caracteristicas-delos-millennials-y-loscentennials/527174

Soto, I. O., \& Ríos, C. P. (2016). Comportamiento y experiencia de consumo desde la interconexión e interactividad de la World Wide Web: un recorrido teórico. I+ D Revista de Investigaciones, 8(2), 35-45.

Stanton, W. (2008). Fundamentos de Marketing. México: Mc. Graw Hill. 